\title{
Revista Española de Geriatría y Gerontología
}

www.elsevier.es/regg

\section{CARTAS CIENTÍFICAS}

\section{Uso de aplicaciones relacionadas con salud en dispositivos móviles y su asociación con comorbilidades en adultos mayores}

\section{Use of health-related applications on mobile devices and their association with comorbidities in older adults}

Durante los últimos años las redes sociales y las aplicaciones para dispositivos móviles (APP) han revolucionado la comunicación y facilitado las actividades diarias. Ello es reflejado en el creciente número de usuarios de smartphones y tablets ${ }^{1}$. Asimismo, según The Pew Research Center's Internet E'American Life Project, el 18\% de adultos mayores (AM) son propietarios de un Smartphone ${ }^{2}$. Actualmente, debido al crecimiento demográfico respecto a la población adulta mayor, el número de comorbilidades se encuentra en crecimiento y ha resultado ser un reto importante para el manejo, cuidado y seguimiento de la salud.

En este contexto las aplicaciones para dispositivos móviles relacionadas con la salud (APPRS) se están convirtiendo en herramientas importantes para el automanejo de enfermedades y en gestión de medicamentos ${ }^{3}$, así como una estrategia útil en asesoramiento y prevención en salud de AM, destacando las relacionadas con la detección y prevención de caídas y asesoramiento funcional $/ \operatorname{cognitivo}{ }^{4}$.

No obstante, existe poco conocimiento sobre el uso de APPRS y su asociación con comorbilidades en AM, pues la mayor parte de información se centra en describir al personal de salud como usuario principal de las APPRS, y no se ha encontrado suficiente evidencia con respecto al rol del AM como usuario. En relación con ello, corrientes de salud pública están intentando forjar políticas de "paciente experto» en este grupo etario, donde la autogestión de la salud depende mucho de las herramientas que tenga el paciente para mantener una adecuada calidad de vida y buen control de enfermedades ${ }^{5}$. Es importante evaluar y cuantificar la asociación entre el uso de APPRS y comorbilidades en AM, y así contribuir con información novedosa sobre un tema poco estudiado sobre prevención en salud 4 .

Como parte de un estudio de cohortes en el Servicio de Geriatría del Centro Médico Naval, se preguntó por el tipo de APP (mensajería, búsqueda de información, juego, noticias y redes sociales) que utilizaban los participantes usuarios de dispositivos móviles (tablets/smartphones), factores sociodemográficos, sexo, edad, educación y comorbilidades (hipertensión, diabetes, depresión, enfermedad renal, enfermedad pulmonar obstructiva crónica, artrosis, osteoporosis, enfermedad tiroidea, anemia, sobrepeso/obesidad y dislipidemias). Además se preguntó: "¿Utiliza alguna APP para consultas o monitorización de su salud?». La base cuenta con datos de 1.277 participantes mayores de 59 años, de los cuales se excluyeron a 515 por tener algún tipo de trastorno neurocognitivo, 300 por déficit auditivo/visual y 164 por presentar enfermedades neurológicas (enfermedad cerebro-vascular, Parkinson, demencia), obteniendo 271 participantes. Se realizó un modelo de regresión logística para calcular OR y determinar la asociación entre uso de APPRS y el número de comorbilidades, considerando significativo un valor de $\mathrm{p}<0,05$.

Se encontró que el $15,87 \%$ de la población utilizaba alguna APPRS, de los cuales el 22,31\% fueron mujeres y el 18,98\% tenía un grado de instrucción alto (tabla 1). Este resultado fue similar al del proyecto The Pew Research Center ${ }^{2}$, donde el 70\% de AM que utilizaba APPRS tenía un alto grado de instrucción. De la misma manera, en un estudio realizado en Estados Unidos se evidenció un mayor uso de APP en mujeres en cuanto a la búsqueda de información sobre salud ${ }^{6}$. Ello indicaría que el uso de APPRS estaría influenciado por distintos factores socioeconómicos en países de bajos recursos.

En cuanto al análisis de múltiples variables, se determinó que no hubo influencia de la edad en el uso de APPRS, y que el uso de APP de juegos, búsqueda de información y noticias incrementaban la probabilidad de usar una APPRS. Ello fue similar al estudio One Health in Social Networks and Social Media ${ }^{7}$, donde la presencia de APPRS es limitada entre los usuarios de redes sociales. Encontramos que el uso de APP de entretenimiento se asoció con menor uso de APPRS; no encontramos estudios con información similar con respecto a este hallazgo.

Si bien un mayor número de comorbilidades se asocia con mayor necesidad de uso de APP de búsqueda de información de salud, existe escasa evidencia en relación con el uso de APPRS por parte de los AM. En relación con ello, en el estudio PLATINO se evaluó a pacientes con enfermedad pulmonar obstructiva crónica, donde se determinó que el número de comorbilidades influyó en la búsqueda activa de información ${ }^{8}$. Otro estudio realizado en AM evidenció una asociación significativa en cuanto a enfermedades crónicas, pero resaltaron la pobre adherencia a uso de APPRS como limitación para lograr un impacto en la salud pública ${ }^{9}$.

Finalmente, se concluye que el número de comorbilidades influye en el uso de APPRS, independientemente del sexo, la edad, el grado de instrucción y el uso de otras APP. Se recomiendan estudios prospectivos del tema para determinar causalidad y contribuir a un manejo adecuado de la salud en AM, tomando en consideración las APPRS como una herramienta potencialmente útil. 
Tabla 1

Factores asociados al uso de APPRS

\begin{tabular}{|c|c|c|c|c|c|c|c|c|c|}
\hline \multirow[t]{2}{*}{ Variable } & \multirow[t]{2}{*}{$\mathrm{N}(\%)$} & \multirow{2}{*}{$\begin{array}{l}\text { Uso de APP } \\
\text { relacionadas con } \\
\text { salud } \\
\mathrm{n}=43(15,87 \%)\end{array}$} & \multirow{2}{*}{$\begin{array}{l}\text { No uso de APP } \\
\text { relacionadas con } \\
\text { salud } \\
\mathrm{n}=228(84,13 \%)\end{array}$} & \multicolumn{3}{|c|}{ Modelo crudo } & \multicolumn{3}{|c|}{ Modelo ajustado } \\
\hline & & & & OR & IC $95 \%$ & Valor de $\mathrm{p}$ & OR & IC $95 \%$ & Valor de $\mathrm{p}$ \\
\hline \multicolumn{10}{|l|}{ Edad } \\
\hline > 80 años & 77 & $11(14,29)$ & $66(85,71)$ & 1 & & & 1 & & \\
\hline 71-80 años & 89 & $11(12,36)$ & $78(87,64)$ & 0,84 & $(0,34-2,08)$ & 0,4 & 0,92 & $(0,91-1,17)$ & 0,4 \\
\hline 60-70 años & 105 & $21(20,00)$ & $84(80,00)$ & 1,5 & $(0,68-3,33)$ & 0,3 & 1,1 & $(0,81-1,13)$ & 0,2 \\
\hline \multicolumn{10}{|l|}{ Sexo } \\
\hline Masculino & 159 & $18(11,32)$ & $141(88,68)$ & 1 & & & 1 & & \\
\hline Femenino & 112 & $25(22,32)$ & $87(77,68)$ & 2,25 & $(1,16-4,36)$ & $\mathbf{0 , 0 2}$ & 1,03 & $(1,07-1,17)$ & 0,045 \\
\hline \multicolumn{10}{|l|}{ Educación } \\
\hline$<11$ años de estudios & 134 & $17(12,69)$ & $117(87,31)$ & 1 & & & 1 & & \\
\hline$\geq 11$ años de estudios & 137 & $26(18,98)$ & $111(81,02)$ & 1.61 & $(0,82-3,13)$ & 0,06 & 1,14 & $(0,88-1,24)$ & 0,3 \\
\hline \multicolumn{10}{|l|}{ Uso de APP } \\
\hline No & & 84 & $14(16,67)$ & $70(83,33)$ & 1 & & & 1 & \\
\hline Mensajería & 187 & $29(15,51)$ & $158(84,49)$ & 0,92 & $(0,46-1,84)$ & 0,8 & 0,94 & $(0,91-1,06)$ & 0,1 \\
\hline No & & 192 & $11(5.73)$ & $181(94.27)$ & 1 & & & 1 & \\
\hline Búsqueda de información & 79 & $32(40,51)$ & $47(59,49)$ & 11,2 & $(5,26-23,87)$ & $\mathbf{0 , 0 0 0 1}$ & 1,16 & $(1,09-1,26)$ & $\mathbf{0 , 0 1}$ \\
\hline No & & 30 & $24(80,00)$ & $6(20.00)$ & 1 & & & 1 & \\
\hline Juegos/entretenimiento & 241 & $19(7,88)$ & $222(92,12)$ & 0,02 & $(0,01-0,06)$ & 0,0001 & 0,7 & $(0,29-0,90)$ & $\mathbf{0 , 0 1}$ \\
\hline No & & 214 & $10(4,67)$ & $204(95,33)$ & 1 & & & 1 & \\
\hline Noticias & 57 & $33(57,89)$ & $24(42,11)$ & 28,05 & $(12,30-63,96)$ & $\mathbf{0 , 0 0 0 1}$ & 2,34 & $(2,16-4,39)$ & $\mathbf{0 , 0 0 0 1}$ \\
\hline No & & 63 & $12(19,05)$ & $51(80,95)$ & 1 & & & 1 & \\
\hline Redes sociales & 208 & $31(14,90)$ & $177(85,10)$ & 0,74 & $(0,36-1,55)$ & 0,4 & 0,88 & $(0,71-1,09)$ & 0,4 \\
\hline \multicolumn{10}{|l|}{ Número de comorbilidades } \\
\hline Ninguna & 41 & $2(4,88)$ & $39(95,12)$ & 1 & & & 1 & & \\
\hline Solo una & 97 & $8(8,25)$ & $89(91,75)$ & 1,75 & $(0,36-8,63)$ & 0,5 & 1,03 & $(0,80-2,08)$ & 0,1 \\
\hline Dos o más & 133 & $33(24,81)$ & $100(75,19)$ & 6,44 & $(1,47-28,11)$ & $<0,0001$ & 2,03 & $(1,21-2,19)$ & $\mathbf{0 , 0 1}$ \\
\hline
\end{tabular}

APP: aplicaciones de teléfonos inteligentes; OR: odds ratio.

En negrita se expresan datos con significación estadística.

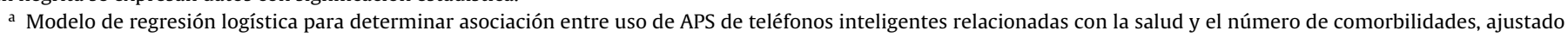
por edad, sexo, educación y uso de otras APP.

\section{Agradecimientos}

A mi familia y al Dr. Runzer.

\section{Bibliografía}

1. Phillips A, Thornbory G. A guide to using smartphones and tablets in occupational health [Internet]. Continuing professional development, Occupational Health. 2014. Disponible en: http://www.personneltoday.com/hr/ a-guide-to-using-smartphones-and-tablets-in-occupational-health/

2. Fox S, Duggan M. Main findings [Internet]. Pew Research Center: Internet, Science \& Tech. 2012 [consultado 27 Ene 2017]. Disponible en: http://www.pewinternet.org/2012/11/08/main-findings-6/

3. Sánchez López MA, Fernández Alemán JL, Toval A, Carrillo de Gea JM. Teléfonos inteligentes para la tercera edad: una revisión de aplicaciones móviles de salud. Rev Costarr Salud Pública, 2014:24:30-42.

4. Bert F, Giacometti M, Gualano MR, Siliquini R. Smartphones and health promotion: A review of the evidence. J Med Syst. 2014;38:9995.

5. Mayorga-Álvarez JH, Montoya-Restrepo LA, Montoya-Restrepo I, SánchezHerrera B, Sandoval-Salcedo NM. Metodología para analizar la carga financiera del cuidado familiar de una persona con enfermedad crónica. Rev Cienc Biomed. 2015;6:96-106.

6. Bietz MJ, Bloss CS, Calvert S, Godino JG, Gregory J, Claffey MP, et al. Opportunities and challenges in the use of personal health data for health research. J Am Med Inform Assoc. 2015;858:1-8.

7. Mekaru SR, Brownstein JS. One health in social networks and social media. Rev Off Int Epizoot. 2014;33:629-37.
8. Varela L, Victorina M, Montes de Oca M, Halbert R, Muiño A Tálamo C et al Comorbidities and health status in individuals with and without COPD in five Latin American Cities: The PLATINO Study. Arch Bronconeumol. 2013;49:468-74.

9. Ancker J, Witteman H, Hafeez B, Provencher T, Van de Graaf M, Wei E. You get reminded you're a sick person: Personal data tracking and patients with multiple chronic conditions. J Med Internet Res. 2015;17:e202.

Cristina Torres-Mallma ${ }^{a}, *$, Fernando M. Runzer-Colmenares ${ }^{\mathrm{b}, \mathrm{c}}$ y José F. Parodi ${ }^{b}$

a Facultad de Medicina Humana, Universidad de San Martín de Porres, Lima, Perú

${ }^{\mathrm{b}}$ Centro de Investigación del Envejecimiento (CIEN), Facultad de Medicina Humana, Universidad de San Martín de Porres, Lima, Perú ' Servicio de Geriatría, Centro Médico Naval, Callao, Perú

* Autor para correspondencia.

Correo electrónico: cristina_torres_95@hotmail.com (C. Torres-Mallma).

https://doi.org/10.1016/j.regg.2017.04.002

0211-139X/

(C) 2017 SEGG. Publicado por Elsevier España, S.L.U. Todos los derechos reservados. 